\title{
Remote Measurement and Shape Reconstruction of Surface-breaking Fatigue Cracks by Laser-Line Thermography
}

\author{
Jinxing Qiu ${ }^{1}$, Cuixiang Pei ${ }^{1,2^{*}}$, Yang Yang ${ }^{1}$, Rongbang Wang ${ }^{1}$, Haochen Liu $^{3}$ and Zhenmao \\ Chen $^{1 *}$ \\ ${ }^{1}$ Shaanxi ERC of NDT and Structural Integrity Evaluation, \\ State Key Laboratory for Strength and Vibration of Mechanical Structures, \\ Xi'an Jiaotong University, Xi'an, 710049, China. \\ ${ }^{2}$ State Key Laboratory of Digital Manufacturing Equipment \& Technology, Wuhan, 430074, China. \\ ${ }^{3}$ School of Aerospace, Transport and Manufacturing, Cranfield University, Cranfield, UK.
}

\begin{abstract}
In this paper, a method for remote measurement and shape reconstruction of fatigue cracks by using laser-line thermography (LLT) technique is developed. A new feature parameter derived from the LLT signals is proposed to evaluate the length and estimate the general inner profile of a surface-breaking fatigue crack. An inversion analysis scheme based on conjugate gradient optimization algorithm is then applied to reconstruct the detailed inner profile and dimension of the crack. The reconstruction results with both numerically simulated LLT signals and experimental signals proved the feasibility of proposed inversion scheme and remote LLT method. Based on methods of this paper, not only the size of cracks can be quantitatively evaluated, but the inner profile is also reconstructed for cracks in different shapes from the LLT signals.
\end{abstract}

Key words: Laser-line thermography; Fatigue crack; Quantitative non-destructive evaluation; Inverse problem; Crack reconstruction

\section{Nomenclature}

$\begin{array}{ll}T & \text { Temperature } \\ t & \text { Time } \\ \rho & \text { Density } \\ c & \text { Thermal capacity } \\ \lambda & \text { Thermal conductive coefficient } \\ q & \text { Heat flux density } \\ \delta & \text { Crack profile indicator } \\ A_{l}, A_{2} & \text { Temperature values along two sides of the crack } \\ u, v & \text { Adjustment parameters of } \delta \\ a, b & \text { Coordinates of two crack tips } \\ c & \text { Center of the crack } \\ d & \text { Depth of the crack } \\ \Delta T & \text { Temperature difference between two sides of the crack } \\ x & \text { Crack parameter vector } \\ \psi & \text { Residual between measured values and simulated values } \\ \alpha & \text { Step size factor in iteration }\end{array}$

\footnotetext{
${ }^{*}$ Corresponding authors.

Email address: pei.cx@mail.xjtu.edu.cn (C. Pei), chenzm@mail.xjtu.edu.cn (Z. Chen)
} 


$\begin{array}{ll}\beta & \text { Conjugate direction } \\ P & \text { Updating direction } \\ g & \text { Gradient vector } \\ \text { NDT\&E } & \text { Nondestructive Testing and Evaluation } \\ \text { ECT } & \text { Eddy Current Testing } \\ \text { DCPD } & \text { Direct Current Potential Drop } \\ \text { FEM } & \text { Finite Element Method } \\ \text { LIT } & \text { Laser Infrared Thermography } \\ \text { LLT } & \text { Laser-line Thermography } \\ \text { CG } & \text { Conjugate Gradient } \\ \text { GSS } & \text { Golden Section Search }\end{array}$

\section{Introduction}

The characterization of fatigue cracks is a challenging task for non-destructive testing and evaluation (NDT\&E) techniques. Recently, with the increasing requirement of maintenance and life prediction of key industrial structures, the shape reconstruction and quantitative evaluation of crack size in structural components is getting more important in the NDT of critical structures such as nuclear power plants, bridges and turbine blades. For most of the NDT methods, only the surface characteristic of crack can be intuitively obtained while the internal information such as the crack depth cannot be obtained directly. The quantitation of crack needs to be accomplished with a signal processing or data analyzing procedure. Among them, one of a promising approach is the inverse analysis, i.e., solving an inverse problem to get quantitative defect information from NDT signals. The inverse analysis using a modelbased optimization method or an artificial intelligence approach has been studied by many researchers for the reconstruction of the surface breaking cracks in different NDT fields, e.g., eddy current testing (ECT) and direct current potential drop (DCPD) method ${ }^{[1-4]}$. By using the inverse analysis, quantitative measurement of crack depth and shapes can be realized even for natural cracks such as stress corrosion cracks.

Laser infrared thermography (LIT) has proven its ability to locate and image surface cracks with features such as remote and intuition. As a fully non-contact inspection method, it has the advantages of high efficiency, being deployable remotely and being suitable for automation. The study objects of LIT contain the laser heating method ${ }^{[5-7]}$, thermal image processing ${ }^{[8-10]}$, and the defect characterizations ${ }^{[11-13]}$. Until now, several studies have been conducted on characterization of surface cracks by using the LIT method ${ }^{[14-18]}$. The main idea of these studies is to evaluate the crack-caused asymmetries of the surface temperature distribution based on image processing. Therefore, evaluation results based on these methods highly depend on the quantitative parameters used in the processing algorithm, which may be affected by the material homogeneity, surface conditions and environment. In general, the quantitative evaluation of surface cracks using LIT signals is still at primary stage as the accuracy and stability of LIT based method are still not satisfied. Besides, current studies mainly concentrate on the artificial crack which usually has a rectangle shape. However, in practical applications the cracks may be in arbitrary shapes although their analytical model can be simplified in a thermodynamic problem.

In a general sense, the quantitative measurement of surface cracks by a non-contact thermographic method means the evaluation of 3D geometric dimensions of the crack. In this spirit, the problem can be decomposed into the quantitation of crack length, width, depth, location and inner profile of boundaries by the data analyzing of an ensemble of thermographic data. The measurement methodology of crack location and length have been 
well reported in former studies ${ }^{[5-9]}$. Combining with image processing algorithms, the crack length, width and location can be directly determined with high accuracy as such information is intuitively illustrated in processed thermal images. The crack width is less important in the quantitation because the width is much small than other dimensions and does not give big influence on the crack evolution. From this viewpoint, the crack depth and inner profile are key parameters in the quantitative evaluation of surface cracks. Principally, such a problem can be solved through reconstruction of the unknown crack information from the inspection signals, which is a typical inverse problem.

In this work, an inverse scheme is adopted and implemented to provide a precise and stable tool for quantitative measurement of surface cracks from LIT signals. First, a crack modelling method is proposed to simplify and parameterize the crack dimensions and shapes in order to study cracks in different shapes. Although the crack shapes are complex in practical application, they can be approximately parameterized based on a rectangle or an elliptical crack model. Second, a new feature parameter which not only is sensitive to crack depth but also can directly indicate crack shape is proposed to improve the reconstruction accuracy and inversion robustness. Third, an inversion scheme based on an improved conjugate gradient (CG) algorithm is developed to predict optimized crack parameters. Finally, the proposed technique is validated through reconstruction cases with use of both simulated and experimental signals.

\section{Method of crack shape identification and numerical analysis}

\subsection{Principle for crack sizing with laser-line thermography}

Among the LIT methods, laser-line thermography (LLT) which utilizes an elliptical line laser as heating source, provides a fast and instantaneous way for the detection of surface cracks. By the crack imaging and extraction process mentioned in paper [6-9], the crack length and location can be measured with relative high accuracy in one testing. Furthermore, a long continuous line laser is generated in LLT nearby the whole crack area, which enables to provide massive information and indication of crack dimensions in whole crack length.

The principle of LLT for crack inspection is illustrated in Fig. 1. During inspection, a line laser beam is excited nearby the crack to heat the inspection target. Subsequently, thermal waves are generated and then propagate to both sides of the laser line. A surface crack that is close to the laser heated region will perturb the lateral heat flow and produce an unsymmetrical temperature distribution in the direction parallel to crack. The blockage of heat flow always causes large temperature gradients across the crack and a common image processing method is to compute the spatial derivative which enhances the gradients and thus extracts cracks in images. Moreover, the heat blockage effect in a local region near the crack is highly dependent on the local crack depth. The deeper the crack is, the lower the temperature value is in the opposite side of the crack, and the larger the temperature gradient is. Theoretically, for a uniform heating source, the distribution of temperature gradient along the direction parallel to crack length can directly reflect the shape and internal profile of the crack. In this way, the inspection and characterization of surface crack can be realized with LLT. However, as most of the laser in practical applications is in a Gaussian elliptical shape at the spatial domain, it is difficult to find a completely uniform line laser source.

In order to solve above problems and realize the quantitative evaluation of crack size by LLT, a new feature parameter that can minimize the influence of laser heating source and give an enhanced indication to the crack shape is proposed in this work. Fig. 2 shows a typical thermal image obtained from LLT testing, which clearly illustrates the temperature difference across the two sides of crack. Then this new feature based indicator can be defined as: 


$$
\delta=\frac{A_{1}-A_{1}^{\prime}+u}{A_{2}-A_{2}^{\prime}+v}
$$

where $A_{1}$ and $A_{2}$ are temperature values along the lines across the crack, $A_{1}$ ' and $A_{2}$ ' are line values at the frame before heating, $u$ and $v$ are adjustment parameters in order to avoid the division of small values. For the experimental conditions, $u=0.1$ and $v=0.1$ are well adapted as shown hereafter. By analyzing the distribution of $\delta$, crack shape is expected be preliminary identified. The performance of $\delta$ will be discussed later in 2.4 .
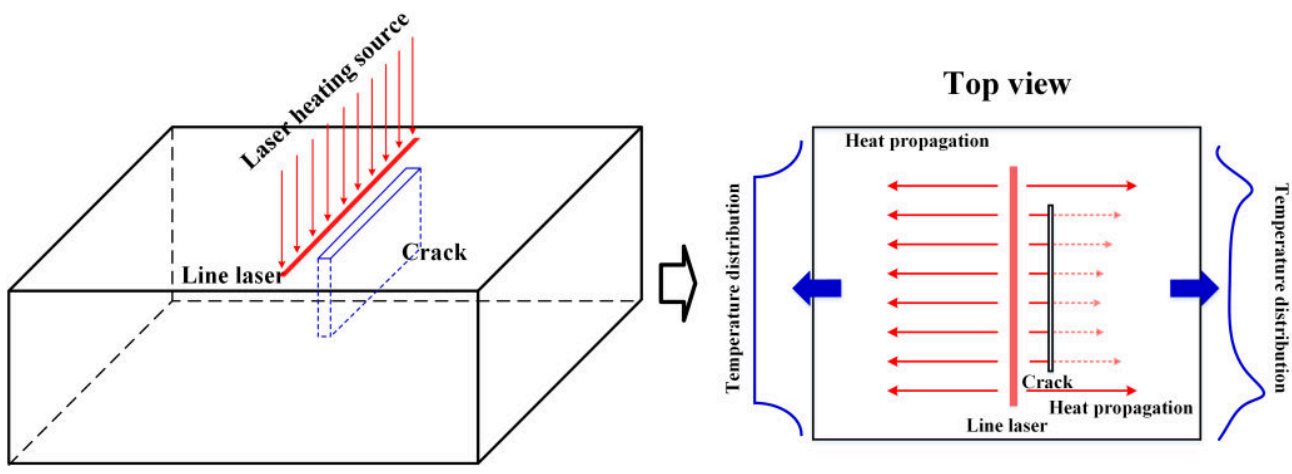

Fig. 1 Principle of LLT for Surface Crack Inspection
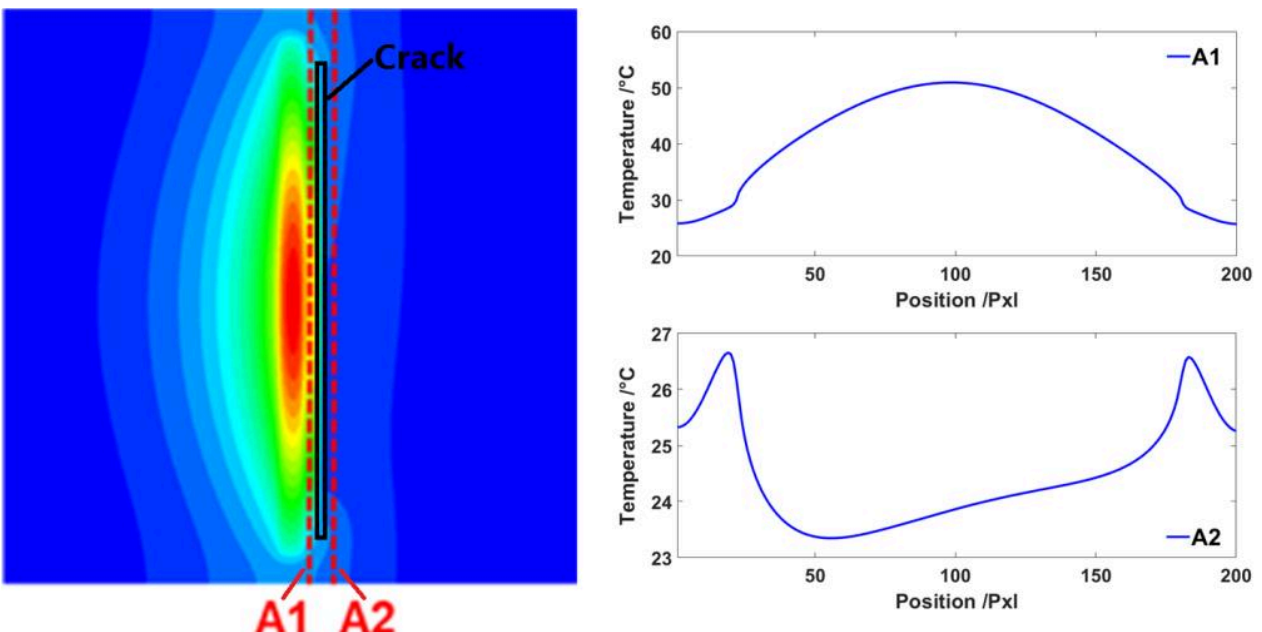

Fig. 2 Definition of crack shape indicator. A1 and A2 represent temperature values along the lines across crack.

\subsection{Modeling of fatigue cracks in different shapes}

To describe the precise surface geometric and actual inner profile is far too difficult for some natural cracks especially the corrosion types, e.g., the stress corrosion cracks, because of their complex profile and internal contact characteristics. Fortunately, for fatigue crack the problem is more distinct as it can be simplified to a half-elliptical shape because the fatigue crack is usually initiated from a small surface defect and propagated to depth and length directions ${ }^{[19-21]}$. In this work, in view of the natural fatigue crack, two crack models are adopted, i.e., crack models of cross section in rectangle shape and half quasi-elliptical shape respectively. This assumption is based on the fact that in IRT the detailed inner profile of crack has weak influence on surface temperature signals and in practice the depth information is much more significant to the safety assessment.

The two crack models are shown in Fig. 3. The longitudinal cross section of the elliptical crack model can be described as a combination of two quarter of ellipse. The two parts share the same center and the depth represents the deepest crack depth located at the center. For the 
coordinate system illustrated in Fig. 3 (a), the crack parameters to describe the crack dimensions are the length $(b-a)$, center position $(|c|)$ and depth $(-d)$, and the crack region in $y$ axis can be defined by

$$
0 \geq y \geq\left\{\begin{array}{lll}
d \sqrt{1-\frac{(x-c)^{2}}{(a-c)^{2}}} & \text { if } \quad x<c \\
d \sqrt{1-\frac{(x-c)^{2}}{(b-c)^{2}}} & \text { if } \quad x \geq c
\end{array}\right.
$$

Meanwhile the rectangle crack model is adopted to study a general case. In this model the crack is considered as a cuboid of a rectangle longitudinal cross section and thin width, and the crack parameters to describe the crack dimensions are the length $(b-a)$ and the depth $(-d)$. Similarly, the crack region in $y$ axis can be defined by

$$
0 \geq y \geq d
$$

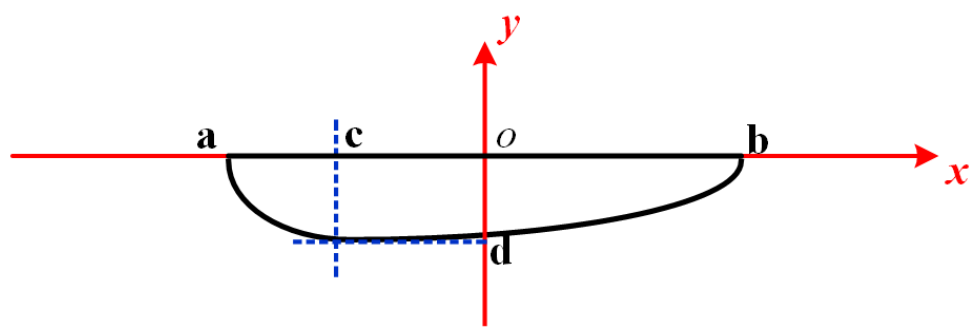

(a) Quasi-elliptical crack model

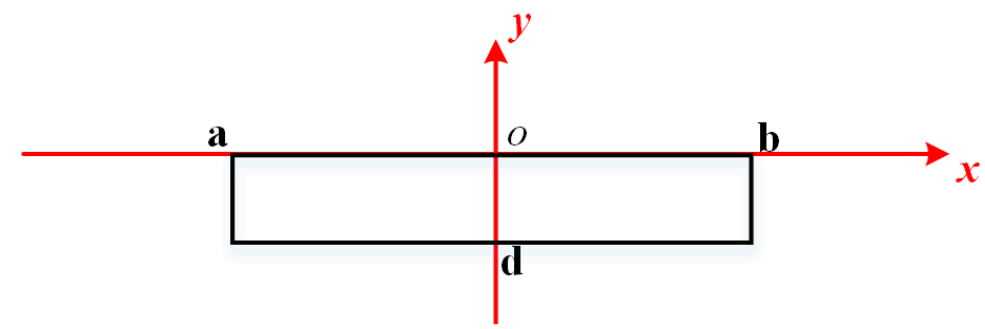

(b) Rectangle crack model

Fig. 3 Parametric model of cracks in different shape

\subsection{Numerical analysis of LLT signals}

In order to make a reliable inverse analysis, a forward analysis method for simulating LLT signals with high accuracy is indispensable. The forward problems related to the LLT method follow the governing equations of the transient thermal conduction problem.

$$
\rho \mathrm{c} \frac{\partial \mathrm{T}}{\partial \mathrm{t}}=\frac{\partial}{\partial \mathrm{x}}\left(\lambda_{x} \frac{\partial \mathrm{T}}{\partial \mathrm{x}}\right)+\frac{\partial}{\partial \mathrm{y}}\left(\lambda_{y} \frac{\partial \mathrm{T}}{\partial \mathrm{y}}\right)+\frac{\partial}{\partial \mathrm{z}}\left(\lambda_{z} \frac{\partial \mathrm{T}}{\partial \mathrm{z}}\right)
$$

where $\rho$ is the density, $c$ is the thermal capacity, $\lambda_{x}, \lambda_{y}, \lambda_{z}$ are the thermal conductive coefficients in $x, y$, and $z$ direction, $T$ is the temperature, $\mathrm{t}$ is the time. Considering the heat flux is applied on the top surface in a LLT problem, the boundary condition of the temperature field can be expressed as

$$
\left.\left(\lambda_{x} \frac{\partial T}{\partial x} n_{x}+\lambda_{y} \frac{\partial T}{\partial y} n_{y}+\lambda_{z} \frac{\partial T}{\partial z} n_{z}\right)\right|_{s}=q_{s}(x, y, z, t)
$$

where $n_{\mathrm{i}}(i=x, y, z)$ is the component of normal vector in $x, y$, and $z$ direction, and $q_{\mathrm{s}}$ is the laser heat source. 
In this study, as we consider the crack may have complex geometry, the analytical solution is very difficult to be solved for the temperature field. Compared with the analytical method, the numerical method has better adaptability and accuracy, although it needs more computer resources. Therefore, the finite element method (FEM) is employed to solve the Eq. (4) and to calculate the surface temperature distribution corresponding to cracks of different shape and dimensions. A numerical simulation code developed by authors is adopted in all of the forward modeling considered herein ${ }^{[18,22-23]}$. The feasibility of the code has been validated by compared with results of both the commercial software and experiments.

A typical simulation model for LLT of a plate with a surface crack is shown in Fig. 4(a), where $A_{0}$ denotes the no-defect region and $A_{\mathrm{c}}$ the region of crack. These two regions are discretized with element of different material, i.e. air for $A_{c}$, and base material for $A_{0}$. The model is uniformly meshed in $x$ and $y$ direction while finer mesh is adopted nearby the top surface in the $z$ direction. The element size is $0.1 \mathrm{~mm} \times 0.1 \mathrm{~mm} \times 0.1 \mathrm{~mm}$ from the first layer to 50th layer. Based on the parametric model of cracks presented in subsection 2.2, the boundary of crack region can be determined by Eq. (2) and (3) and the elements above this boundary are defined as crack elements. An example of the mesh division for an elliptical crack is shown in Fig. 4(b).

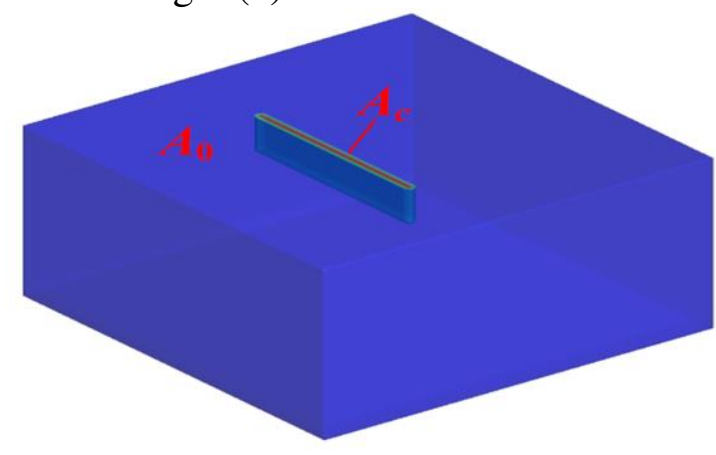

(a)

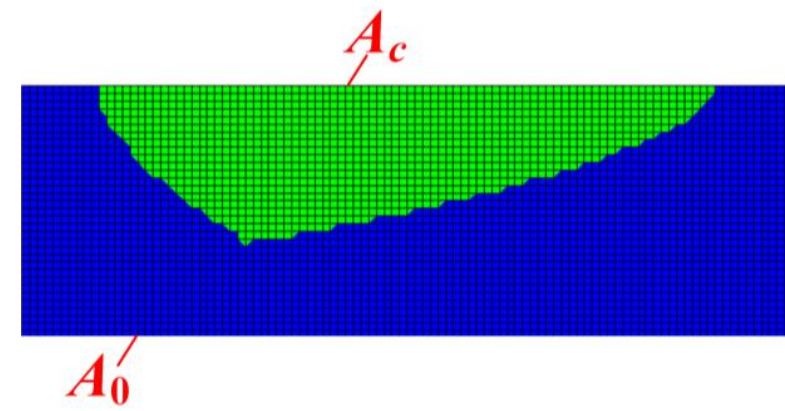

(b)

Fig. 4 Simulation model: (a) 3D model, (b) mesh near crack region

Simulations were firstly conducted with a line laser excited on the surface near crack region and the temperature field on the surface at each time step were obtained. Fig. 5 illustrates the crack models used in three simulation cases: a rectangle crack and two elliptical cracks. The detailed crack dimensions are list in Table I. The corresponding simulation results of the temperature distribution at time $4 \mathrm{~s}$ of above three cases are illustrated in Fig. 6 . According to simulation results, after laser heating source is applied on the specimen surface, the heat flow will propagate to lateral region. As the thermal conductivity in crack region is much lower than that of the base materials, the heat flow is perturbed and rounded across the crack with a time delay. In this manner, a temperature discontinuity can be observed in the temperature field over the crack line. The perturbation caused by the crack differs along the crack for different crack shapes because of the variation in depth. Thus, heat blockage effect in shallow region of crack is smaller than that of deep region. As shown in Fig. 7, the temperature distribution along the right side of the crack follows the variation of crack depth, i.e., the crack depth profile is possibly to be revealed by the heat blockage effect.

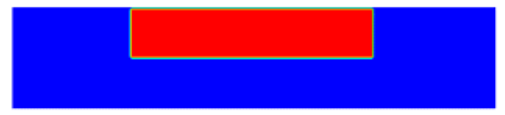

(a)

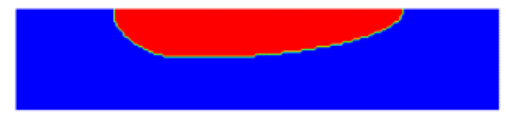

(b)

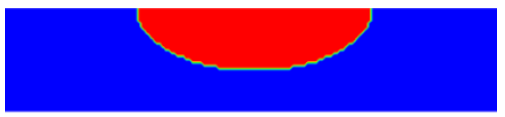

(c)

Fig. 5 Cracks in simulation cases: (a) crack 1: rectangle crack; (b) crack 2: oblique elliptical crack; (c) crack 3: half-elliptical crack. 


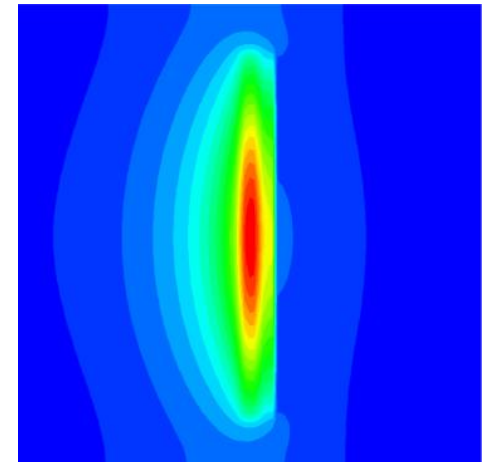

(a)

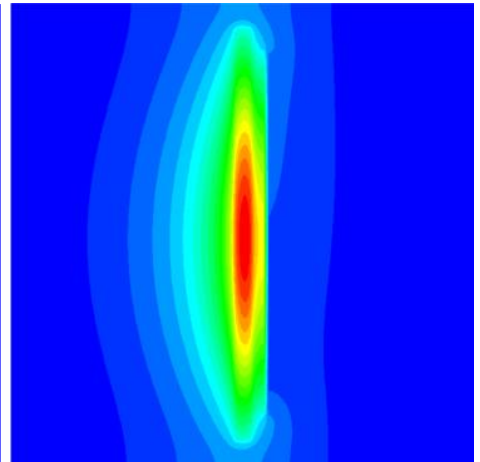

(b)

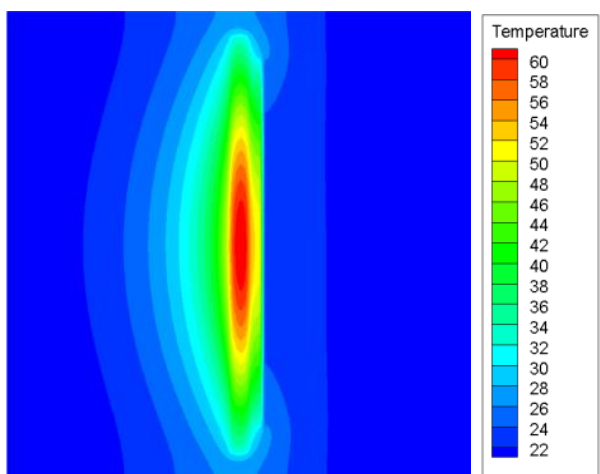

(c)

Fig. 6 Surface temperature field in three simulation cases: (a) crack 1; (b) crack 2; (c) crack 3.

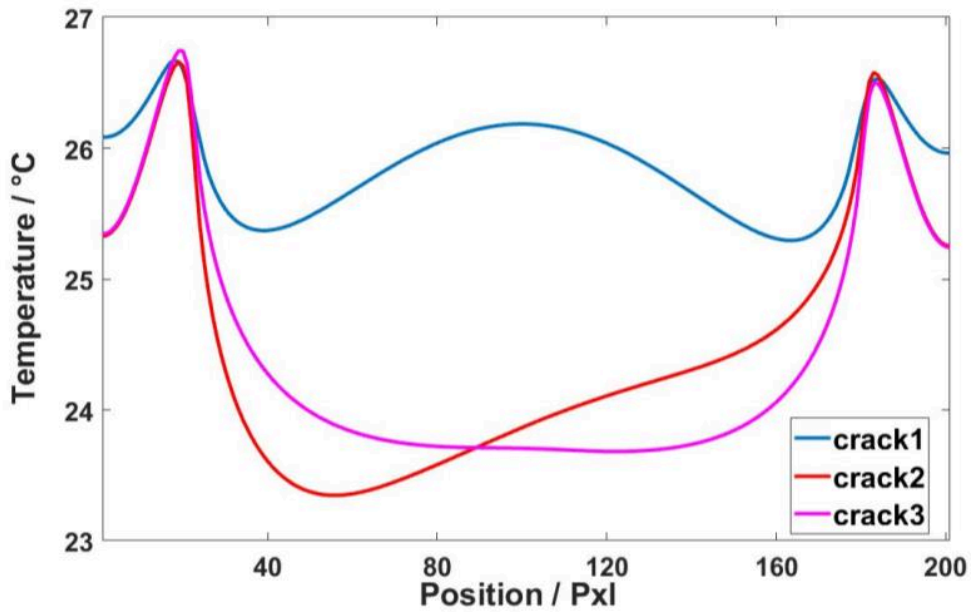

Fig. 7 Temperature distribution along the right side of crack.

Table I Crack sizes in simulation cases.

\begin{tabular}{lcccc}
\hline & Opening $(\mathrm{mm})$ & Length $(\mathrm{mm})$ & Center $(\mathrm{mm})$ & Depth $(\mathrm{mm})$ \\
\hline Crack 1 & 0.1 & 16 & - & 2 \\
Crack 2 & 0.1 & 16 & -4 & 2 \\
Crack 3 & 0.1 & 16 & 0 & 2 \\
\hline
\end{tabular}

\subsection{Results of general inner profile estimation of the crack}

The variation of heat blockage effect appeared in Fig. 7 is inconspicuous as this phenomenon is mainly in the temperature field on the right side of crack which is far away from the laser heating source. Especially the irregular temperature distribution caused by the surface patterns and inhomogeneous laser density distribution in the practical measurements may easily mask the indication of this effect. Therefore, the choice of characteristic signal to representing the temperature field has a great impact on the final reconstruction results. The characteristic signal should be sensitive to defect profile but robust to noise. The conventional characteristic signal of LIT, such as the temperature difference between two sides of the crack $(\Delta T)$ is extracted as shown in Fig. 8. It is clear that this characteristic signal is not suitable for the crack reconstruction as the change due to different crack is hardly to be witnessed. This may because temperature variation caused by the uneven heating is much more significant than that caused by crack shape for line laser source of Gauss distribution. Therefore, it is necessary to find a feature parameter which can directly reflect the crack shape.

As mentioned in subsection 2.1, the new feature parameter may show its advantage for crack reconstruction. Fig. 9 shows the distribution of parameter $\delta$ for the three cracks. It can be seen that this indicator is much more sensitive to the depth compare with $\Delta T$. Therefore, the crack shape can be preliminary identified by reviewing the distribution of $\delta$. 


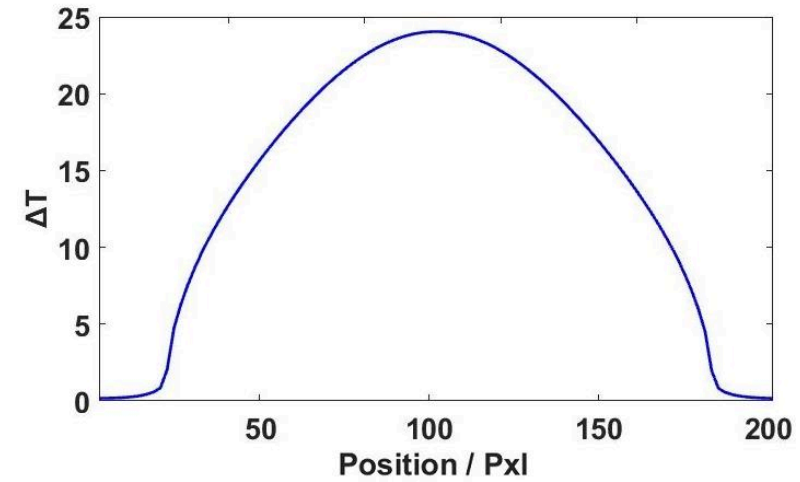

(a) crack 1

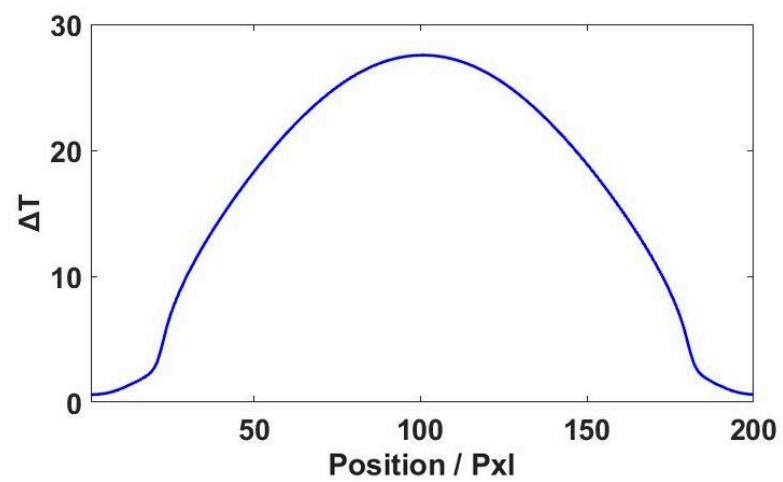

(b) crack 2

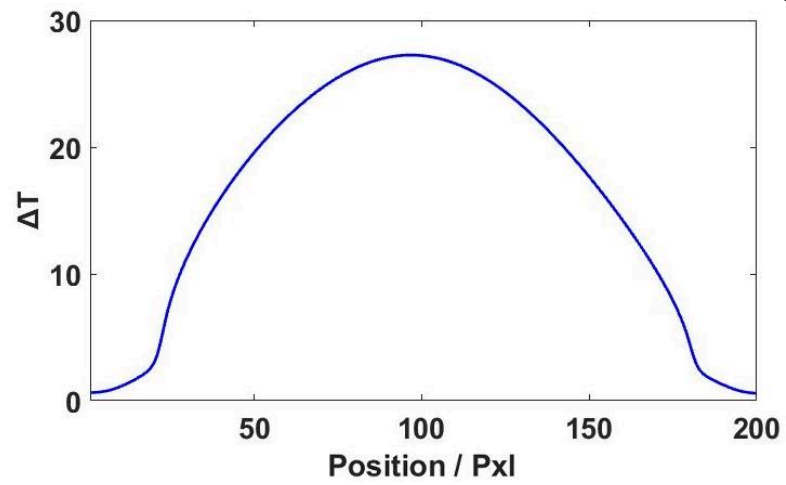

(c) crack 3

Fig. 8 The distribution of $\Delta T$ along crack direction.

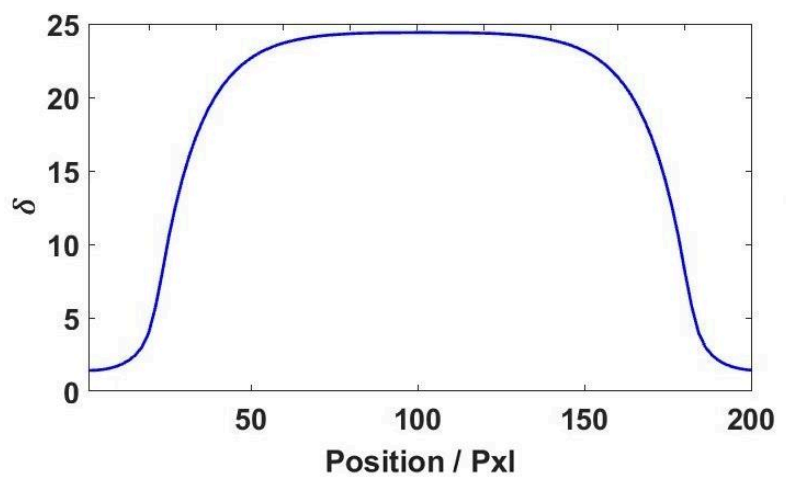

(a) crack 1

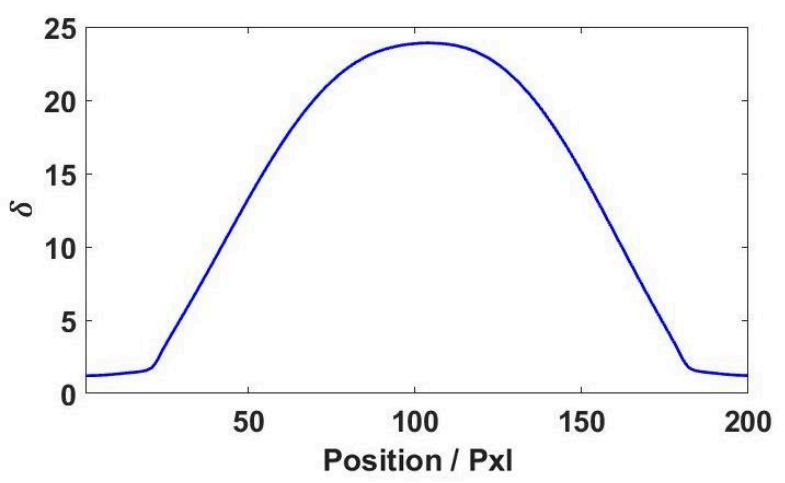

(b) crack 2

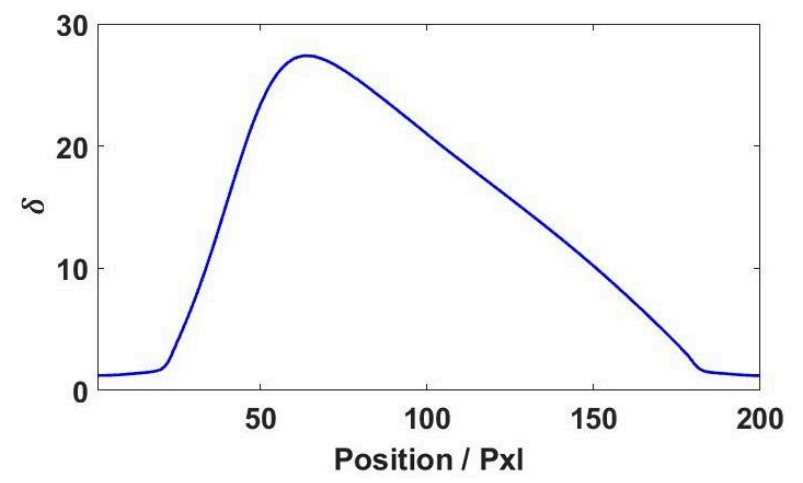

(c) crack 3

Fig. 9 The distribution of $\delta$ along crack direction.

\section{Inversion methodology for crack shape reconstruction with LLT}

3.1 Inverse analysis scheme for crack reconstruction 
As an inverse problem, reconstruction of crack parameters from temperature signals can be expressed as an optimization problem, i.e., to search the optimal parameter vector $\{\boldsymbol{x}\}$ of crack to minimize the error function between the measured temperature signals $\left\{T^{e x p}\right\}$ and the simulated ones $\left\{T^{\operatorname{sim}}\right\}$. The objective function in this optimization problem can be defined by

$$
\psi(\boldsymbol{x})=\sum_{n=1}^{M}\left|T_{n}^{\operatorname{Sim}}(\boldsymbol{x})-T_{n}^{E x p}\right|^{2}
$$

where $M$ is the total number of signal points, $T_{n}{ }^{\operatorname{sim}}(\boldsymbol{x})$ is the temperature at $n$-th signal point when the crack is of parameter $\boldsymbol{x}$. In this work, the conjugate gradient (CG) optimization algorithm is adopted to solve this optimization problem iteratively. The CG optimization method has been successfully applied to reconstruct surface cracks from ECT and DCPD signals ${ }^{[2-4]}$. In a similar way, the CG inverse strategy is updated for the reconstruction of the crack size and inner profile from LIT signals. The key procedure of the CG-based method to solve the inverse problem is the following iteration algorithm to update unknown vector $\boldsymbol{x}$

$$
\boldsymbol{x}_{k+1}=\boldsymbol{x}_{k}+\alpha_{k} \boldsymbol{P}_{k}
$$

where $\boldsymbol{P}_{k}$ is the updating direction of $k$-th iteration which is usually chosen as the direction of the conjugate gradient vector of the objective function

$$
\boldsymbol{P}_{k}= \begin{cases}-\boldsymbol{g}_{k} & (k=1) \\ -\boldsymbol{g}_{k}+\beta_{k} \boldsymbol{P}_{k-1} & (k \geq 2)\end{cases}
$$

where $\beta_{k}$ is a parameter that determine the conjugate direction. In CG method

$$
\beta_{k}=\frac{\boldsymbol{g}_{k}^{T} \boldsymbol{g}_{k}}{\boldsymbol{g}_{k-1}{ }^{T} \boldsymbol{g}_{k-1}}
$$

$\boldsymbol{g}_{k}$ is the gradient vector of $k$-th step vector with its $i$-th element equal

$$
\boldsymbol{g}_{k, i}=\frac{\partial \psi^{k}}{\partial x_{i}}=2 \sum_{n=1}^{M}\left\{\left\{T_{n}^{S i m}\left(\boldsymbol{x}^{k}\right)-T_{n}^{E x p}\right\} * \frac{\partial T_{n}^{S i m}\left(\boldsymbol{x}^{k}\right)}{\partial \boldsymbol{x}_{i}}\right\}
$$

In Eq. (10), the key is how to obtain the gradient values of the residual $\partial T_{n}^{S i m}(\boldsymbol{x}) / \partial \boldsymbol{x}_{i}$. As an explicit analytical solution for the thermal conduction equation is not available for us, the difference scheme was adopted to calculate the gradient values in each iteration step, i.e. to calculate the partial differential value of simulated signals when the selected crack parameter is respectively $\boldsymbol{x}+\Delta \boldsymbol{x}_{i}$ and $\boldsymbol{x}$

$$
\frac{\partial T_{n}^{S i m}(\boldsymbol{x})}{\partial \boldsymbol{x}_{i}}=\frac{T_{n}^{\text {Sim }}\left(\boldsymbol{x}+\Delta \boldsymbol{x}_{i}\right)-T_{n}^{\text {Sim }}(\boldsymbol{x})}{\Delta \boldsymbol{x}_{i}}
$$

The step size factor $\alpha_{k}$ in CG is usually calculated by the following equation

$$
\alpha_{k}=\left(\sum_{n=1}^{M}\left\{T_{n}^{S i m}(\boldsymbol{x})-T_{n}^{E x p}\right\} * \frac{\partial T_{n}^{S i m}(\boldsymbol{x})}{\partial \alpha_{k-1}}\right) / \sum_{n=1}^{M}\left|\frac{\partial T_{n}^{S i m}(\boldsymbol{x})}{\partial \alpha_{k-1}}\right|
$$

In general, crack parameter vector $\{\boldsymbol{x}\}$ consists of the crack position, crack shape and crack dimension parameters. In this work, the inversion is conducted after the crack imaging procedure was performed, i.e., we suppose the crack position and approximated crack length are known. The flowchart for the reconstruction of cracks is illustrated in Fig. 10. For a rectangle crack, only the crack depth is taken as the target parameters for a primary stage and the CG method is adopted to solve the crack depth. For an elliptical crack, the crack center position is also taken as the target parameters in addition to the crack depth. As the two parameters give very different influence on the LLT signals, different inversion scheme is adopted for their reconstruction, i.e., the crack depth is updated by using CG method, while the golden section search (GSS) method is adopted to search the optimum crack center 
parameter. In practice, instead of the temperature signal, the feature parameter $\delta$ along the crack as descripted in subsection 2.3 is adopted as the input signals. As this feature parameter is very sensitive to crack shape, the reconstruction efficiency can be significantly improved.

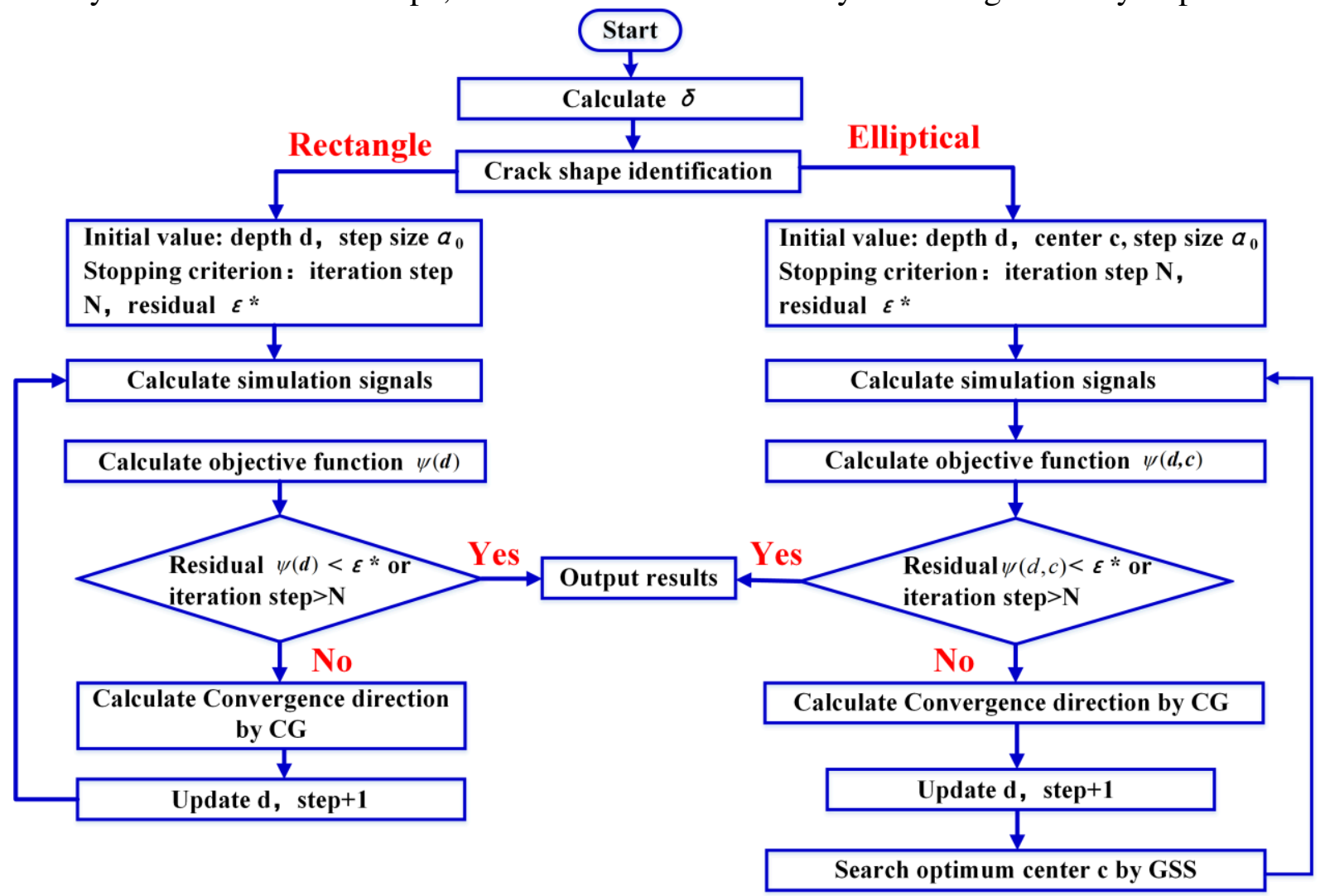

Fig. 10 Flow chart of the crack shape reconstruction method

\subsection{Numerical validation of the inversion scheme}

In order to validate the proposed inversion method, its performance is firstly checked by using simulated LLT signals. The simulation model and laser heating pattern are the same as that mentioned in subsection 2.2. A rectangle crack (crack 1) and an elliptical crack (crack 2) are reconstructed as typical validation cases. To check the robustness of method, $2 \%$ white noise is added into the simulated signals. The detailed reconstruction procedure is as follows:

Step 1: Extract feature parameter $\delta$ from simulated LLT signals and identify the crack shape based on the distribution of $\delta$;

Step 2: Set initial values and terminating criterion. For rectangle crack, initial crack depth is set as $0.1 \mathrm{~mm}$. Minimum residual error is set as 0.01 and maximum iteration step is 10. For elliptical crack, initial depth is set as $0.1 \mathrm{~mm}$ and initial center position coordinate is $0.0 \mathrm{~mm}$. Minimum residual error is set as 0.1 and maximum iteration step is 20;

3) Update crack parameters based on the algorithm shown in Fig.10;

4) Output reconstructed results if terminating condition is triggered.

Fig. 11 and Fig. 12 show the reconstructed results for the two selected cracks respectively. Fig. 11 (a) and (b) present the evolution of crack size and residual error that reveal a good convergence. Fig. 11 (c) and (d) show the comparisons of the true and reconstructed feature signals and crack profiles. The output depth value is $2.0 \mathrm{~mm}$ which is the same as real depth. A similar behavior can be found in Fig. 12 for the case of elliptical crack. The output depth value is $2.0 \mathrm{~mm}$ and the center is $-3.3 \mathrm{~mm}$, comparably the real values are $2.0 \mathrm{~mm}$ and $-4.0 \mathrm{~mm}$. The near-perfect accordance in both crack size and inner profile demonstrates the feasibility of the proposed method and related inversion code. 


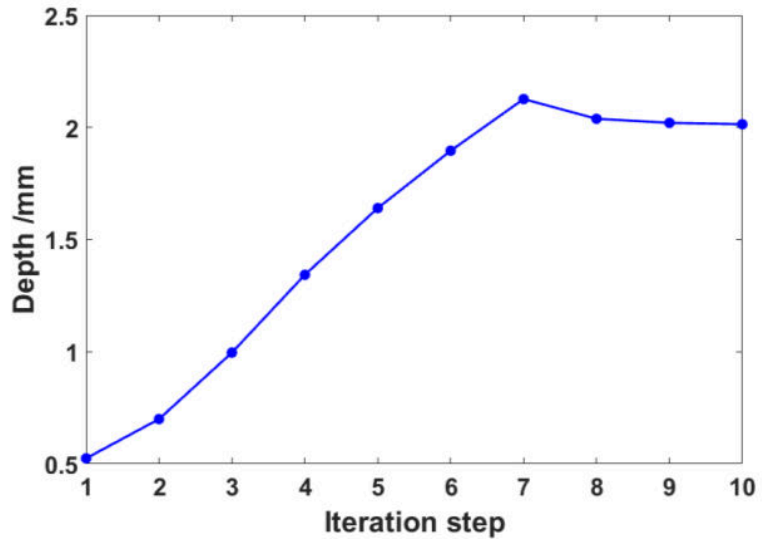

(a)

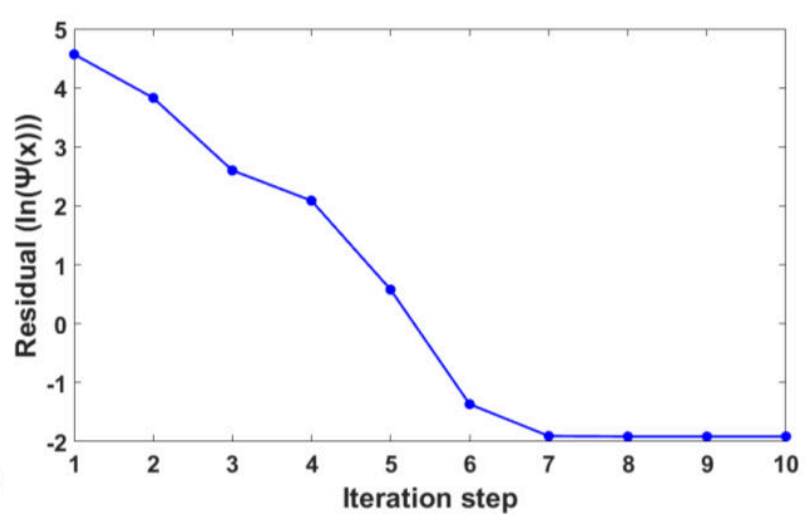

(b)

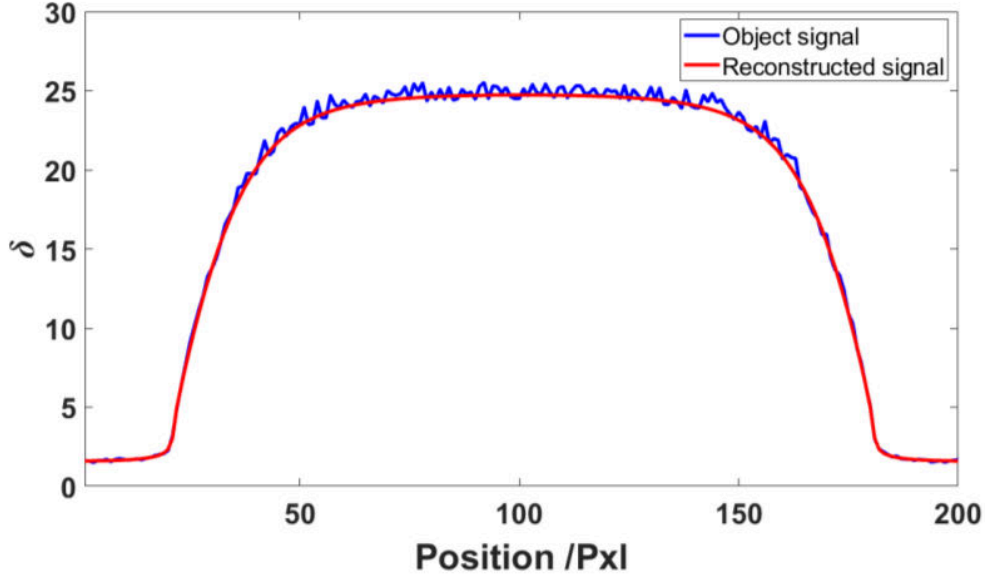

(c)

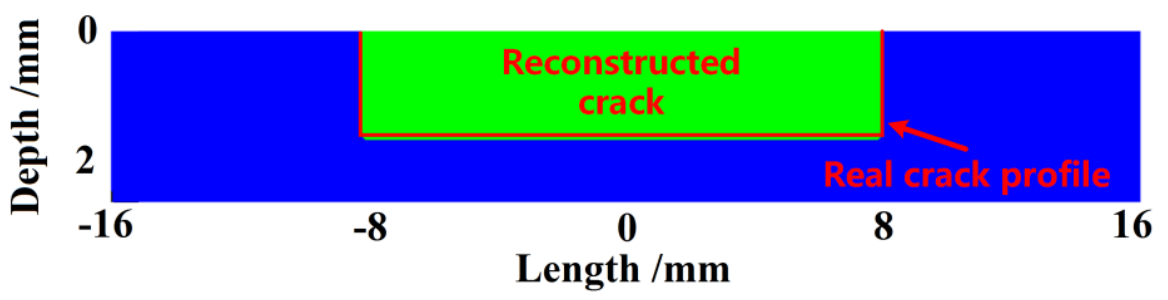

(d)

Fig. 11 Reconstructed results of the rectangle crack: (a) iteration process of the crack depth; (b) iteration process of the logarithmic residual; (c) comparison of the characteristic signals;

(d) comparison of the reconstructed crack profile and real crack profile.

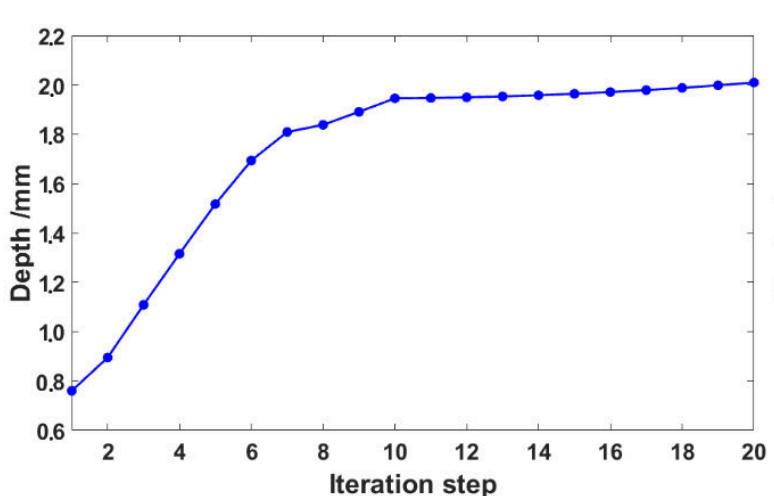

(a)

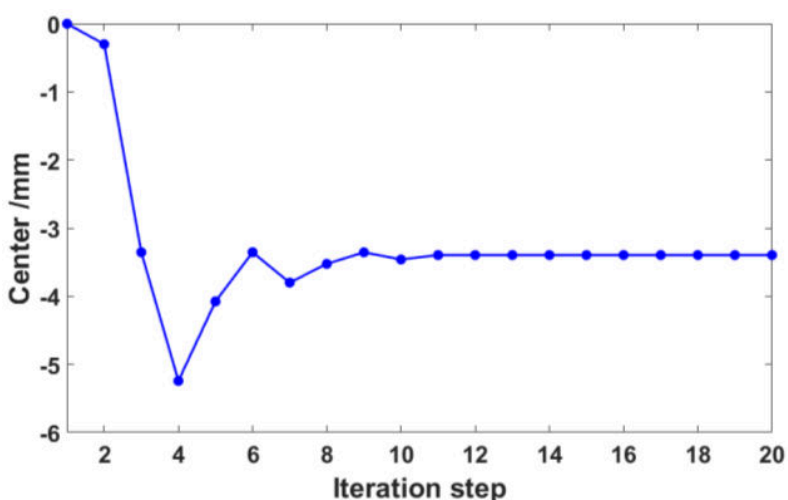

(b) 


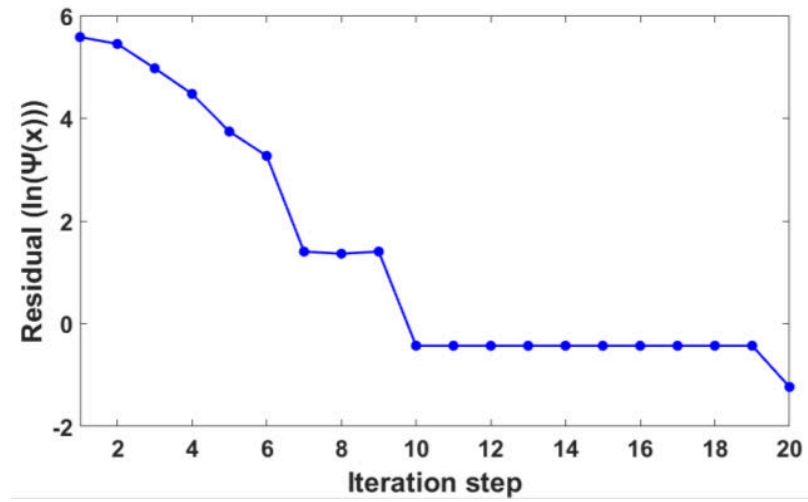

(c)

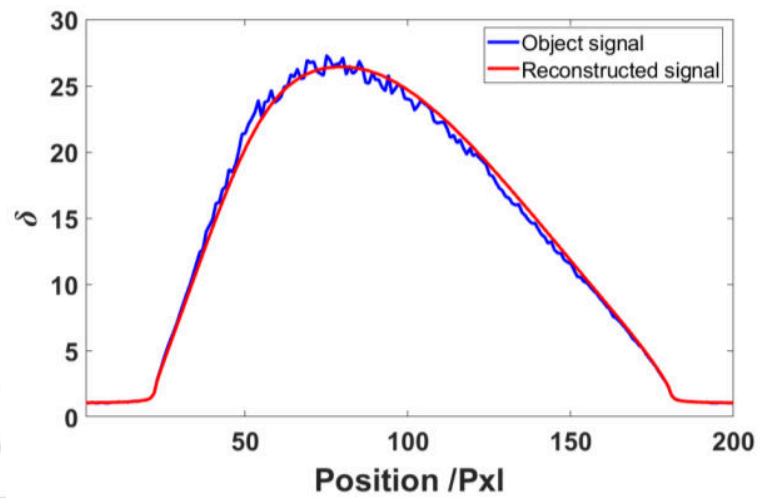

(d)

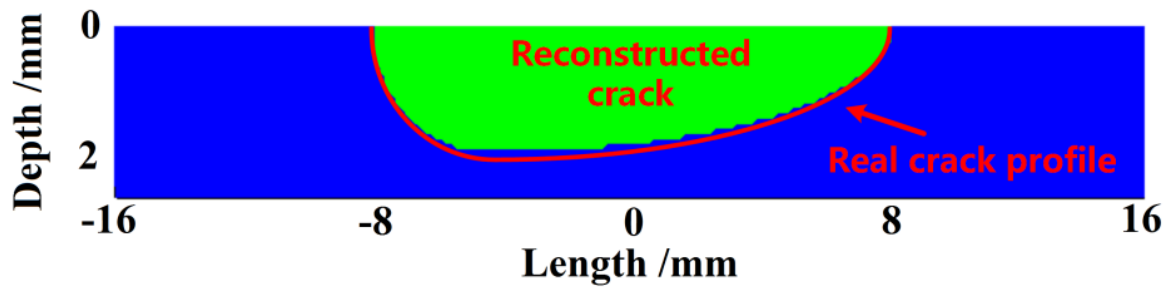

(e)

Fig. 12 Reconstructed results of the elliptical crack: (a) iteration process of the crack depth; (b) iteration process of the crack center; (c) iteration process of the logarithmic residual; (d) comparison of the characteristic signals; (e) comparison of the reconstructed crack profile and real crack profile.

\section{Experimental validation of the inversion scheme}

\subsection{Experimental setup and specimens}

An LLT experimental system for surface crack inspection and measurement is designed as illustrated in Fig. 13. The system is composed of excitation, sensing, data processing and control units. The excitation unit includes an arbitrary waveform generator (AWG), a laser driver, and a CW laser head. The CW laser used in the system has a wavelength of $1064 \mathrm{~nm}$ and a maximum power of $100 \mathrm{~W}$. The sensing, data processing and control units comprise an infrared (IR) camera and a personal computer (including data acquisition and processing software). The established experimental system is shown in Fig. 14. The IR camera used here has a noise equivalent temperature difference of $50 \mathrm{mK}$, a resolution of $640 \times 512$ pixels at a sampling rate of $60 \mathrm{~Hz}$, and a spectral range of 7.5 14 $\mu \mathrm{m}$. While inspecting, a 4 seconds square wave laser pulse was applied to the specimen. The initial round laser beam $(4 \mathrm{~mm}$ in diameter) was expanded by 6 times with an expander and then focused into an elliptical laser beam $(0.8 \mathrm{~mm}$ in width and $24 \mathrm{~mm}$ in length) by a cylindrical lens.

In order to validate the inversion scheme experimentally, experimental signals due to artificial fatigue cracks were measured. In practice, three SUS304 specimen plates with slit crack of different profiles as shown in Fig. 15 were fabricated by using electrical discharge machining technique. The detailed specifications of the crack and plate of the three specimens are shown in Table II, which are denoted as No.1, No.2 and No.3 hereafter. 


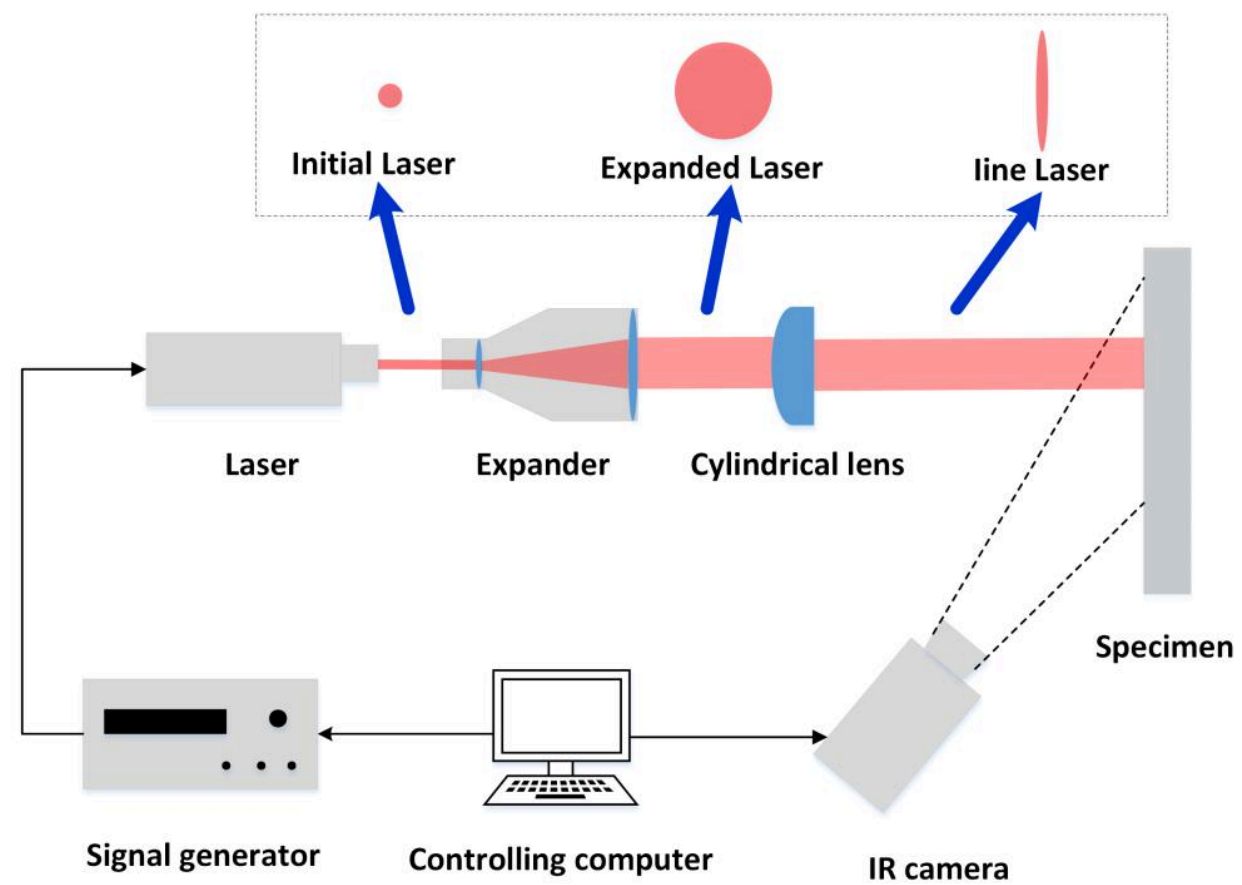

Fig. 13 Schematic of the laser-line thermography experimental system

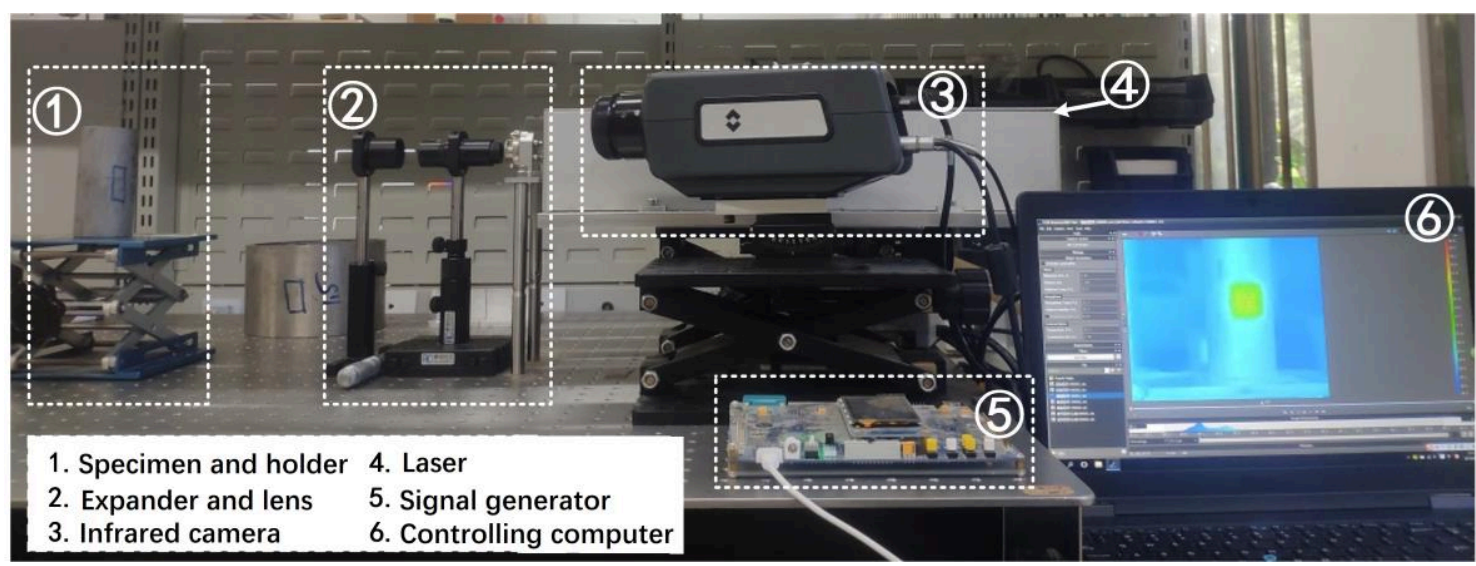

Fig. 14 Experimental setup of the laser-line thermography system.
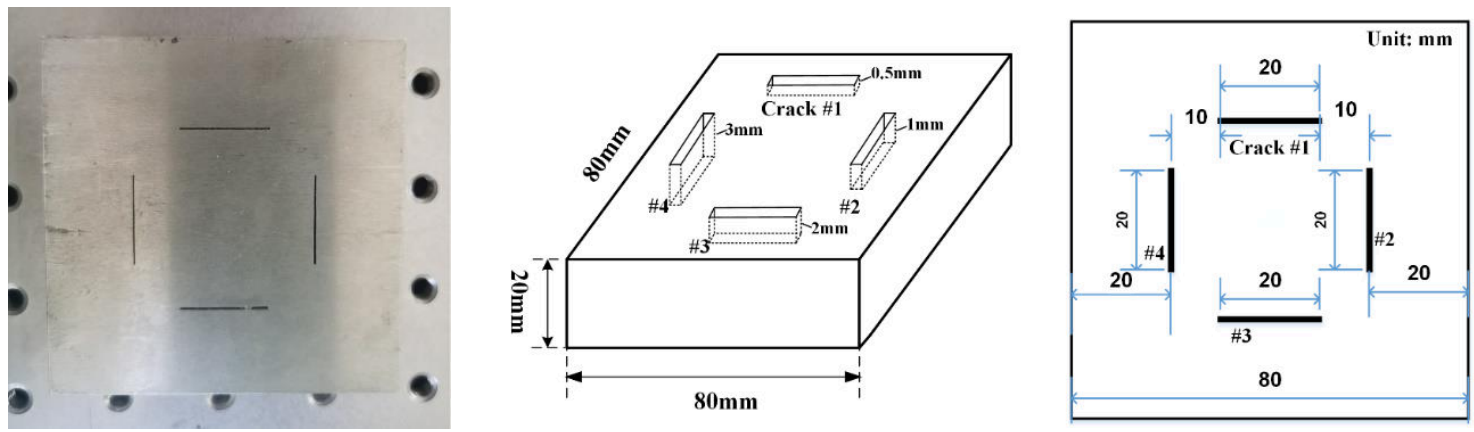

(a) 

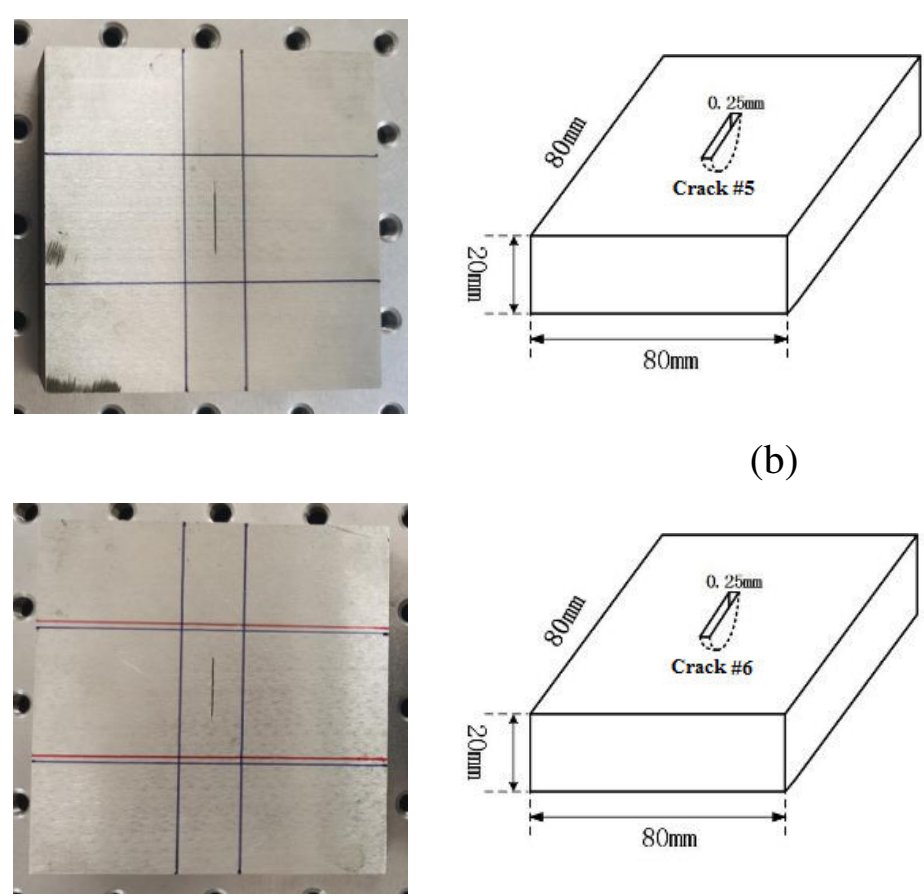

(c)

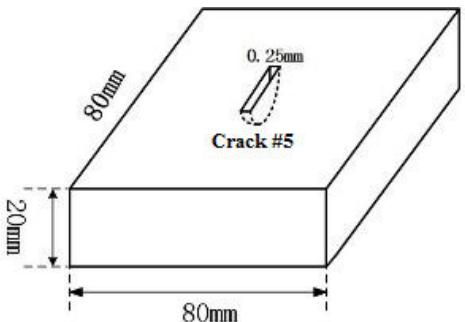

(b)
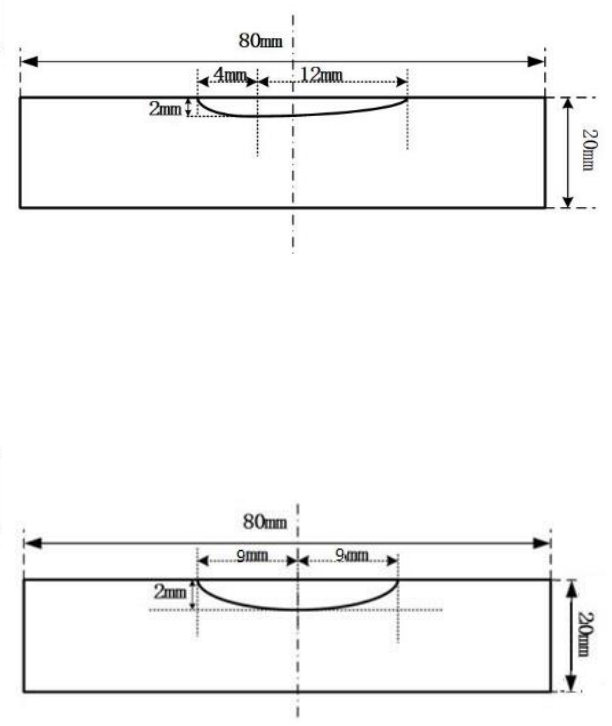

Fig. 15 Photo and schematic of specimens: (a) No.1 specimen; (b) No.2 specimen; (c) No.3 specimen.

Table II Specifications of specimens.

\begin{tabular}{|c|c|c|}
\hline Specimen & Sizes of specimens & Specifications of cracks \\
\hline No. 1 & $\begin{array}{l}\text { plate with } 4 \text { rectangle cracks; } \\
\text { Length: } 100 \mathrm{~mm} \text {; Width: } 100 \mathrm{~mm} \text {; } \\
\text { Thickness: } 20 \mathrm{~mm} \text {. }\end{array}$ & $\begin{array}{l}\text { Length: } 20 \mathrm{~mm} \text {; Width: } 0.25 \mathrm{~mm} \text {; } \\
\text { Depth: } 0.5 \mathrm{~mm}, 1 \mathrm{~mm}, 2 \mathrm{~mm}, 3 \mathrm{~mm} \text {. }\end{array}$ \\
\hline No. 2 & $\begin{array}{l}\text { plate with a quasi-elliptical crack; } \\
\text { Length: } 80 \mathrm{~mm} \text {; Width: } 80 \mathrm{~mm} \text {; } \\
\text { Thickness: } 20 \mathrm{~mm} \text {. }\end{array}$ & $\begin{array}{l}\text { Length: } 16 \mathrm{~mm} \text {; Width: } 0.25 \mathrm{~mm} \text {; } \\
\text { Depth: } 2 \mathrm{~mm} \text {; Center: }-4 \mathrm{~mm} \text {. }\end{array}$ \\
\hline No. 3 & $\begin{array}{l}\text { plate with an elliptical crack; } \\
\text { Length: } 80 \mathrm{~mm} \text {; Width: } 80 \mathrm{~mm} \text {; } \\
\text { Thickness: } 20 \mathrm{~mm} \text {. }\end{array}$ & $\begin{array}{l}\text { Length: } 18 \mathrm{~mm} \text {; Width: } 0.25 \mathrm{~mm} \text {; } \\
\text { Depth: } 2 \mathrm{~mm} \text {; Center: } 0 \mathrm{~mm} \text {. }\end{array}$ \\
\hline
\end{tabular}

\subsection{Reconstruction results with measured signals}

In experiments, the recorded thermal images were firstly processed by subtracting an initial thermal image measured before heating to eliminate the background noise. The feature signal is then extracted from the thermal image for the inverse analysis. With use of the developed inversion code, all the cracks are reconstructed from the measured signals. All numerical calculation was carried out in a workstation with Intel Xeon E5 CPU and the time cost of each iteration was about 3 minutes.

The reconstruction results for rectangle cracks and elliptical cracks are respectively listed in Table III and IV. In Fig. 16 and Fig. 17, true and reconstructed feature signals and crack profiles are comparisons for six cracks. The results show that satisfied inversion results were also obtained and also reveal that the proposed feature parameter $\delta$ is a good indicator for crack profile and the proposed inversion scheme appears to be effective for the reconstruction of surface cracks in different shapes. Compared to the results presented in 3.2 with simulated signals, the error of reconstruction results with measured signals increase to around $15 \%$. The 
main reason of the error is that the simulation conditions were not exact the same with that in experiment. For instance, the laser used in simulation can be set as in ideal round shape while in experiments it is difficult to utilize an ideal laser source. The uniform distribution of laser power density may be changed after expanding with the optical lens.
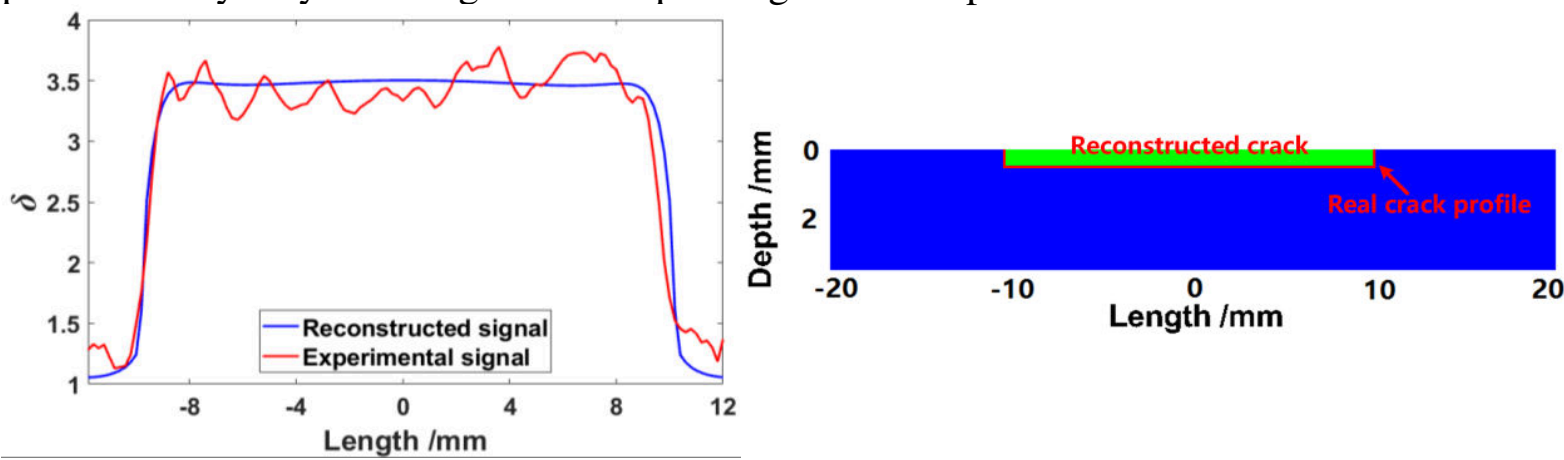

(a) Reconstructed results for Crack \#1
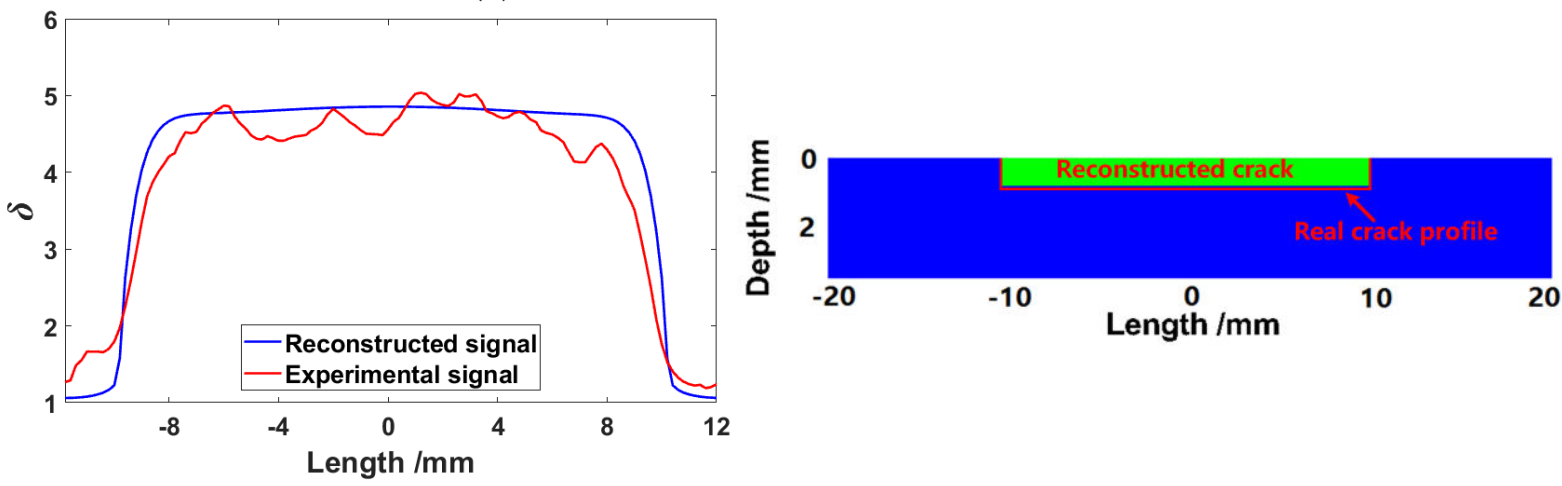

(b) Reconstructed results for Crack \#2
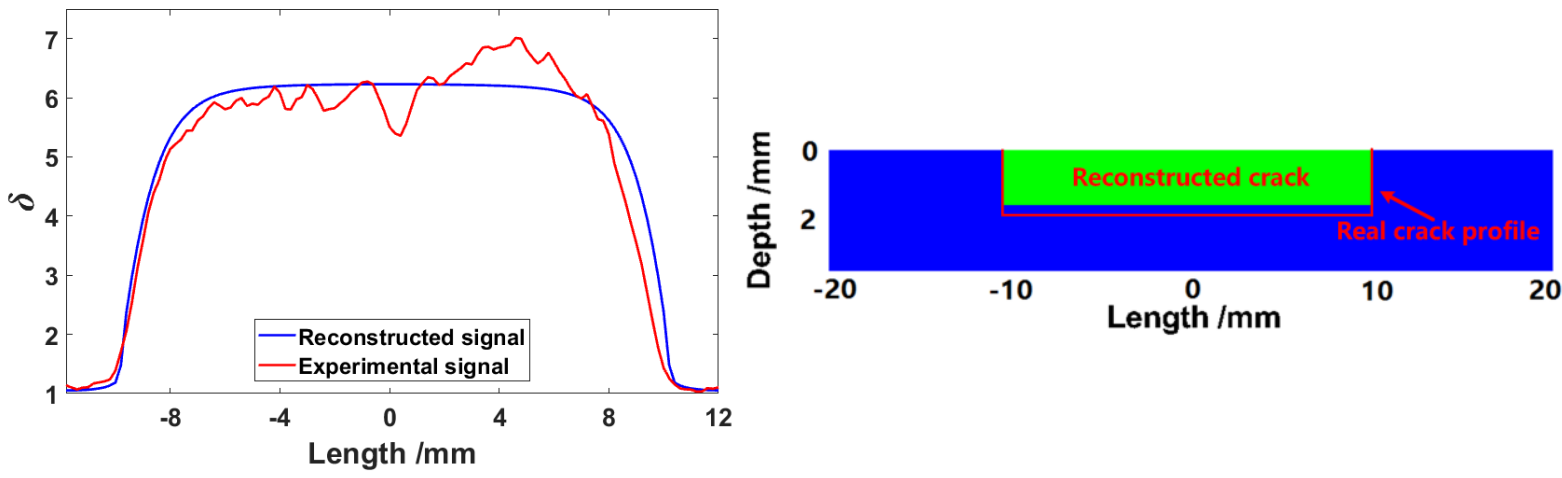

(c) Reconstructed results for Crack \#3
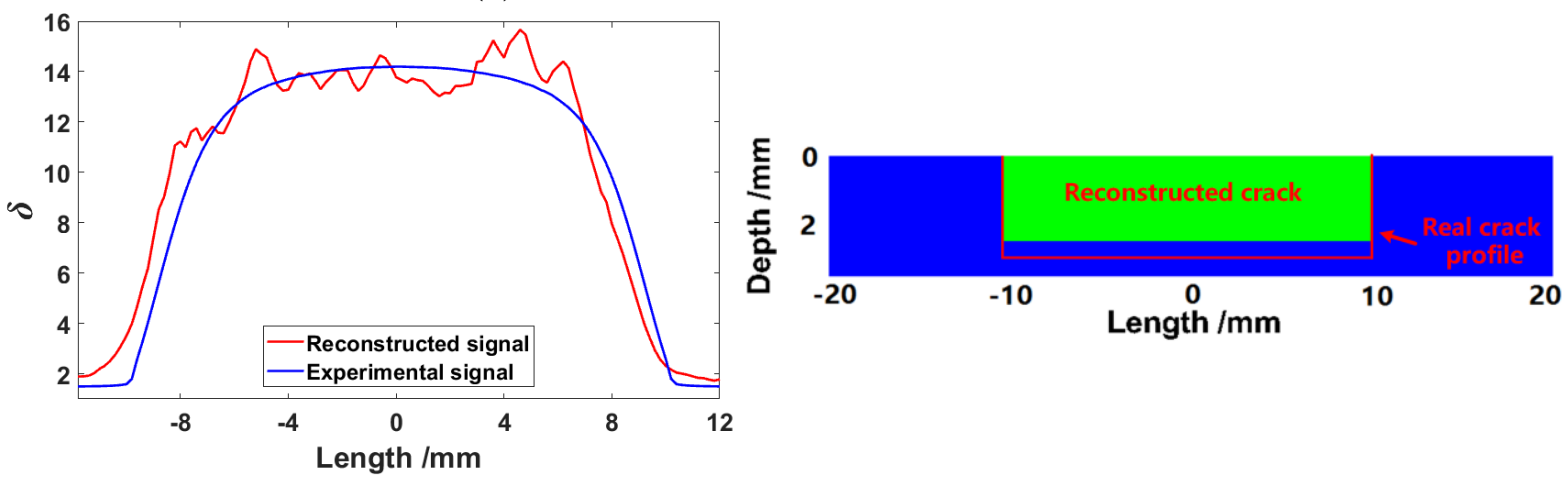

(d) Reconstructed results for Crack \#4

Fig. 16 Reconstructed results of rectangle cracks: reconstructed characteristic signals vs. experimental characteristic signals and reconstructed crack profile vs. real crack profile. 

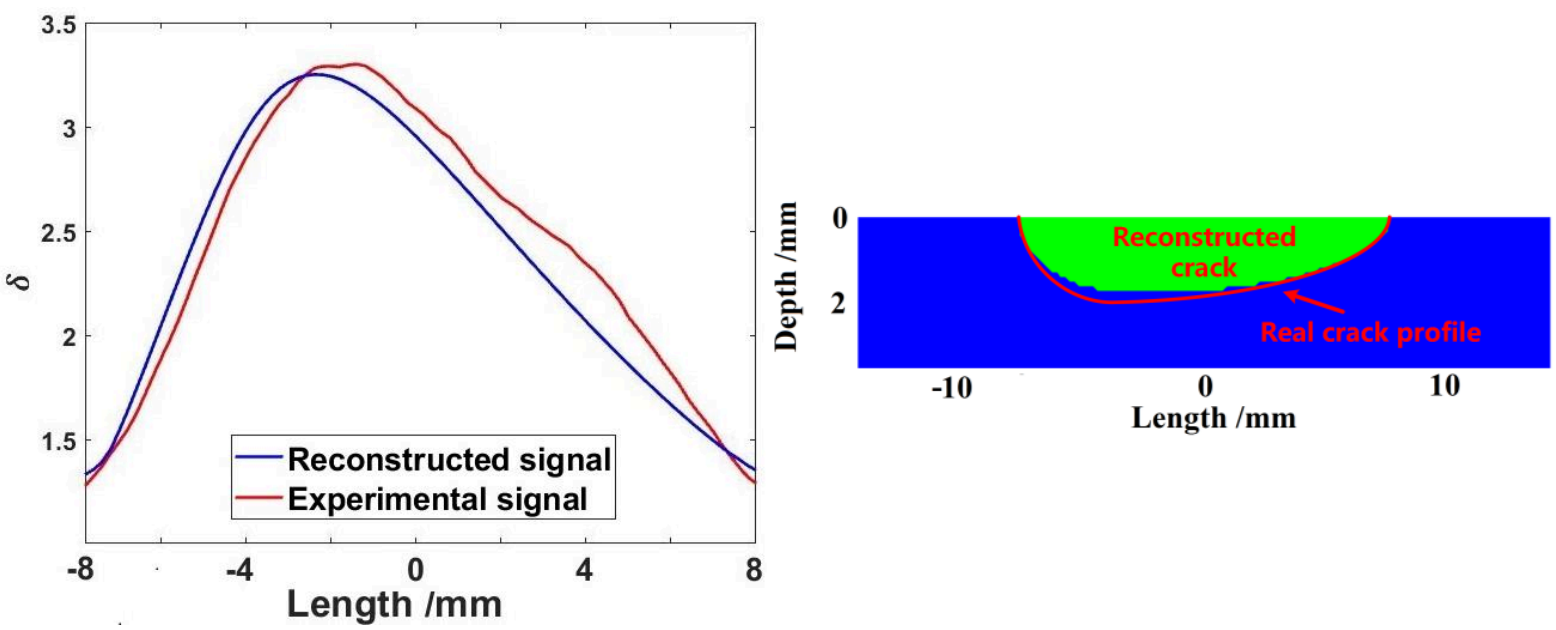

(a) Reconstructed results for Crack \#5
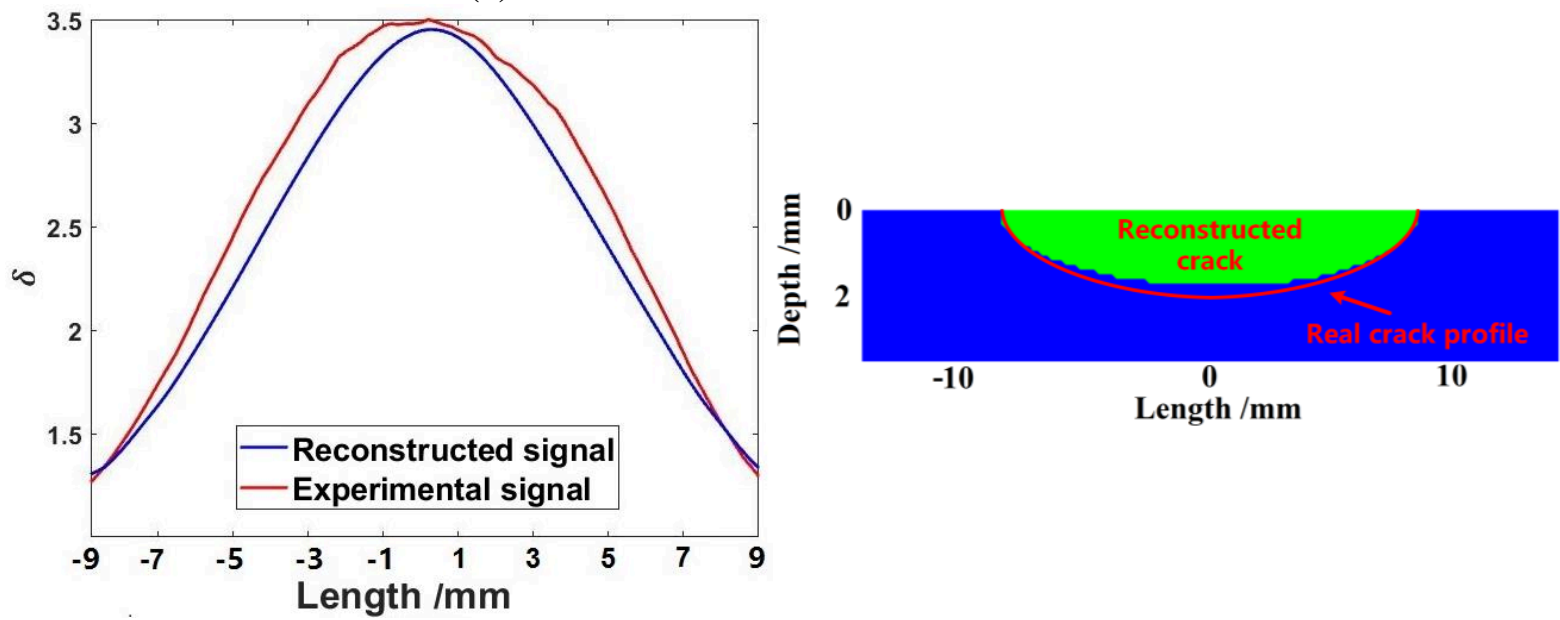

(b) Reconstructed results for Crack \#6

Fig. 17 Reconstructed results for elliptical cracks.

Table III Reconstruction results for rectangle cracks.

\begin{tabular}{cccc}
\hline Crack number & Real depth $(\mathrm{mm})$ & Reconstructed depth $(\mathrm{mm})$ & Error \\
\hline$\# 1$ & 0.5 & 0.5 & $0 \%$ \\
$\# 2$ & 1.0 & 0.9 & $10 \%$ \\
$\# 3$ & 2.0 & 1.7 & $15 \%$ \\
$\# 4$ & 3.0 & 2.6 & $13 \%$ \\
\hline
\end{tabular}

Table IV Reconstruction results for elliptical cracks.

\begin{tabular}{|c|c|c|c|c|c|}
\hline \multirow{2}{*}{\multicolumn{2}{|c|}{ Crack dimensions }} & \multicolumn{2}{|c|}{ \#5 } & \multicolumn{2}{|c|}{ \#6 } \\
\hline & & Value & Error & Value & Error \\
\hline \multirow{2}{*}{$\begin{array}{l}\text { Depth } \\
(\mathrm{mm})\end{array}$} & Real & 2.0 & \multirow[b]{2}{*}{$15.0 \%$} & 2.0 & \multirow[b]{2}{*}{$15.0 \%$} \\
\hline & Reconstructed & 1.7 & & 1.7 & \\
\hline \multirow{2}{*}{$\begin{array}{l}\text { Center } \\
(\mathrm{mm})\end{array}$} & Real & -4.0 & \multirow[b]{2}{*}{$6.9 \%$} & 0 & \multirow[b]{2}{*}{$3.3 \%$} \\
\hline & Reconstructed & -2.9 & & 0.6 & \\
\hline \multirow{2}{*}{$\begin{array}{l}\text { Area } \\
\left(\mathrm{mm}^{2}\right)\end{array}$} & Real & 25.13 & \multirow{2}{*}{$15.0 \%$} & 28.27 & \multirow{2}{*}{$15.0 \%$} \\
\hline & Reconstructed & 21.36 & & 24.03 & \\
\hline
\end{tabular}

It should be noted that all results presented above are under following assumptions: 1) the crack is entirely noncontact in its interior and can be regarded as fulling with air. For real cracks, it may be partially closed and the thermal property in crack is between air and base materials. For such cracks the influence of thermal resistance variation cannot be neglected. 2) 
The inversion analysis was conducted after the crack location and length were clarified. In this way, we can ensure that in each measurement, the line laser used to heat the sample locally can be generated parallel to the crack in a fixed distance and the length of laser is long enough to cover the whole crack. Moreover, the location of laser source is kept constant in each measurement as the distance between laser source and crack significantly influence the measurement results. 3) The surface of specimen is not rough and without much rust and scratches. For objects of which the surface condition is undesirable, proper surface treatment such as polish operation or painting may be desired. The measurement of NDT signals is in laboratory environment and the effects of environmental conditions are inconspicuous compared to other conditions. For the measurement of objects in outdoor environment, the environmental conditions such as humidity, temperature and weather should be taken into consideration before testing.

\section{Conclusions}

In this study, a quantitative reconstruction approach based on a model-based inversion algorithm was proposed for sizing surface-breaking fatigue cracks in different shapes from LLT signals. Compared to conventional method based on calibration, this approach does not require such high cost and time-consuming calibration procedure. In addition, we propose a new feature parameter that is highly sensitive to crack shapes and sizes to enhance the inversion precision. Compared to normally used parameters such as temperature difference, this new feature parameter can directly reflect the crack shape regardless of the uniformity of laser heating source. Furthermore, a hybrid inversion strategy is developed by combining the CG and GSS method to reconstruct crack depth and center respectively. Compared to a conventional inversion strategy which reconstruct multiple crack parameters simultaneously, the proposed method significantly improves the ill-posedness of the inverse problem.

The proposed technique is validated with use of both the simulated and experimental signals to reconstruct unknown crack parameters. The results show that the developed method is well adapted to measure cracks with depth of less than $3 \mathrm{~mm}$. For both the rectangle and elliptical cracks, their shapes and sizes were obtained with relative error of less than $15 \%$ even by using experimental signals, which proved the validity of proposed inversion method and corresponding numerical code.

By the crack parameterization method proposed in this paper we assume that the shape of crack can be approximated as a rectangle or ellipse. In further studies, a more general parameterization model will be taken into investigation in order to extend the application of proposed method to reconstruction of cracks in arbitrary shapes and furtherly improve the accuracy of crack profile reconstruction.

\section{Acknowledgements}

The authors would like to thank the National Natural Science Foundation of China (No. 11927801), National Key R\&D Program of China (2017YFF0209703) and the State Key Lab of Digital Manufacturing Equipment \& Technology (No. DMETKF2018012) for funding.

\section{References}

[1] W. Cheng, S. Kanemoto, I. Komura, et al., "Depth sizing of partial-contact stress corrosion cracks from ECT signals," NDT\&E International, vol. 39, no. 5, pp. 374-383, July 2006.

[2] Z. Chen, K. Aoto, K. Miya, "Reconstruction of cracks with physical closure from signals of eddy current testing," IEEE Trans, Magn., vol. 36, no. 4, pp. 1018-1022, Aug. 2000.

[3] P. Shi, S. Su, Z. Chen, "Overview of Researches on the Nondestructive Testing Method of Metal Magnetic Memory: Status and Challenges," Journal of Nondestructive Evaluation, vol. 39, no. 43, pp. 1-37, May 2020. 
[4] W. Cai, S. Xie, X. Wang, C. Pei, Z. Chen, N. Yusa, "Assessment of Local Conductivity Distribution in Stress Corrosion Crack Region Using Direct Current Potential Drop Method," Corrosion Science, vol. 123, pp. 197-208, July 2017.

[5] R. Yang, Y. He, "Optically and Non-optically Excited Thermography for Composites: A Review," Infrared Physics \& Technology, vol. 75, pp. 26-50, Mar. 2016.

[6] Y. An, J. Yang, S. Hwang, H. Sohn, "Line laser lock-in thermography for instantaneous imaging of cracks in semiconductor chips," Optics and Lasers in Engineering, vol. 73, pp. 128-136, Oct. 2015.

[7] J. Qiu, C. Pei, H. Liu, Z. Chen, and K. Demachi, "Remote inspection of surface cracks in metallic structures with fiber-guided laser array spots thermography," NDT\&E Int., vol. 92, pp. 213-220, Dec. 2017.

[8] Y. An, J. Kim, H. Sohn, "Laser lock-in thermography for detection of surface-breaking fatigue cracks on uncoated steel structures," NDT\&E Int., vol. 65, pp. 54-63, July 2014.

[9] J. Yang, J. Choi, S. Hwang, et al., "A reference-free micro defect visualization using pulse laser scanning thermography and image processing," Measurement Science and Technology, vol. 27, no. 8, July 2016.

[10] S. Holland, J. Renshaw, "Physics-based image enhancement for infrared thermography," NDT\&E Int., vol. 43, no. 5, pp 440-445, July 2010.

[11] W. Jeong, C. Earls, W. Philpot, et al., "Inverse thermographic characterization of optically unresolvable through cracks in thin metal plates," Mechanical Systems and Signal Processing, vol. 27, 634-650, Feb. 2012.

[12] N. Pech-May, A. Oleaga, A. Mendioroz, et al., "Vertical cracks characterization using lock-in thermography: I infinite cracks," Measurement Science and Technology, vol. 25, Sep. 2014.

[13] R. Celorrio, A. Omella, A. Mendioroz, et al., "Advances in Crack Characterization by Lock-In Infrared Thermography," International Journal of Thermophysics, vol. 36, pp. 1202-1207, July 2015.

[14] J. Schlichting, C. Maierhofer, M. Kreutzbruck, "Crack sizing by laser excited thermography," NDT\&E International, vol. 45, no. 1, pp. 133-140, Jan. 2012.

[15] S. Beuve, Z. Qin, J. Roger, et al., "Open cracks depth sizing by multi-frequency laser stimulated lock-in thermography combined with image processing," Sensors and Actuators A: Physical, vol. 247, pp. 494-503, Aug. 2016.

[16] Y. Fedala, M. Streza, J. Roger, et al., "Open crack depth sizing by laser stimulated infrared lock-in thermography," Journal of Physics D Applied Physics, vol. 47, no. 46, Oct. 2014.

[17] C. Boue, S. Hole, "Open crack depth sizing by multi-speed continuous laser stimulated lock-in thermography Measurement Science and Technology," Measurement Science and Technology, vol. 28, no. 6, Apr. 2017.

[18] J. Qiu, C. Pei, H. Liu, Z. Chen, "Quantitative evaluation of surface crack depth with laser spot thermography," International Journal of Fatigue, vol. 101, no. 1, pp. 80-85, Aug. 2017.

[19] D. Broek, "Fatigue crack propagation," Strength of Materials, vol.20, no. 7, pp. 841-853, July 1982.

[20] T. Wang, Z. Lou, "A continuum damage model for weld heat affected zone under low cycle fatigue loading," Eng. Fract. Mech., vol. 37, no. 4, pp. 825-829, Feb. 1990.

[21] T. Wang, "A continuum damage model for ductile fracture of weld heat affected zone," Eng. Fract. Mech., vol. 40, no. 6, pp. 1075-1082, Feb. 1991.

[22] H. Liu, C. Pei, S. Xie, Z. Chen, "Numerical simulation method for IR thermography NDE of delamination defect in multilayered plate," International Journal of Applied Electromagnetics and Mechanics, vol. 52, no. 1, pp. 381-389, Dec. 2016.

[23] H. Liu, S. Xie, C. Pei, J. Qiu, Y. Li, Z. Chen, "Development of a Fast Numerical Simulator for Infrared Thermography Testing Signals of Delamination Defect in a Multilayered Plate," IEEE Transactions on Industrial Informatics, vol. 14, no. 12, pp. 5544-5551, Apr. 2018. 
2020-09-15

Remote measurement and shape reconstruction of surface-breaking fatigue cracks by laser-line thermography

Qiu, Jinxing

Elsevier

Qiu J, Pei C, Yang Y, et al., (2021) Remote measurement and shape reconstruction of surface-breaking fatigue cracks by laser-line thermography. International Journal of Fatigue, Volume 142, January 2021, Article number 105950

https://doi.org/10.1016/j.jifatigue.2020.105950

Downloaded from Cranfield Library Services E-Repository 